\title{
From Silver Plates to Spherical Nanoparticles: Snapshots of Microwave-Assisted Polyol Synthesis
}

\author{
Miquel Torras and Anna Roig*
}

Cite This: ACS Omega 2020, 5, 5731-5738

Read Online

ABSTRACT: The fabrication of silver nanoparticles (Ag NPs) with different sizes by microwave (MW)-assisted synthesis is presented. The fast heating of the MW technique, combined with the possibility to thermally quench the reactions, enabled us to capture snapshots of nucleation and growth processes difficult to accomplish in other techniques. The Ag NPs were synthesized using poly(vinylpyrrolidone) (PVP) through a polyol approach. The effects of the reaction time, the reaction temperatures, and the silver precursor concentration were investigated. The influence of agitation, the PVP concentration, and the initial conditions of the silver precursor was also studied. It is found that at very short reaction times and at low temperatures, polyhedral plates are formed with sizes ca. $300 \mathrm{~nm}$ and large polydispersity. However, by increasing the time or the temperature, a size and shape refinement is observed resulting in $10 \mathrm{~nm}$ spherical NPs with low polydispersity. Mechanistic insights are provided based on the observations extracted from transmission electron microscopy (TEM) and ultraviolet-visible spectros-

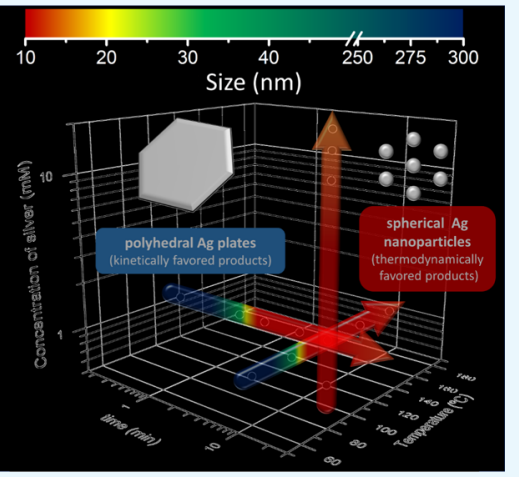
copy (UV-vis). A formation mechanism starting from kinetically favored silver polyhedral plates to thermodynamically favored spherical nanoparticles is proposed. Understanding these effects allowed us to control the particle size and the tuning of Ag NPs ondemand. Moreover, the reproducibility and scalability of the process and the long-term stability of the NPs in aqueous solutions are demonstrated. Finally, we provide a recommendation regarding the use of fresh PVP as a capping and stabilizing agent.

\section{INTRODUCTION}

Every day, nanotechnology is prevailing more in many society domains and industrial sectors such as health, pharmaceutics, food, information technologies, electronics, energy, or the environment. ${ }^{1}$ New applications, devices, and products derived from advances in nanotechnology demand new nanomaterials, specifically, functional nanoparticles (NPs). The migration of these NPs from the laboratory to the market is proceeding at a rather slow pace and needs to be accelerated. Thus, wellunderstood, reproducible, and scalable methods for efficient NP production are needed.

Metal nanoparticles are of particular industrial interest due to their unique properties and applications both in technology and in biomedicine. ${ }^{2-4}$ Their optic, catalytic, electronic, and spectroscopic properties make them interesting in many domains. They are characterized by a localized surface plasmon resonance (LSPR) absorption band. The position of this band strongly depends not only on the type of metal but also on its size, shape, and to a lesser extent, the dielectric environment of the particles. ${ }^{5-7}$ Silver nanoparticles (Ag NPs) are especially interesting due to their antimicrobial properties. ${ }^{8}$ They have been widely used in water and air filtration to eliminate microorganisms ${ }^{9}$ and in the biomedical field because of their intrinsic therapeutic properties. ${ }^{10}$ Moreover, their high electrical conductivity and chemical stability make them the perfect material for inkjets in electronics, photonics, biomarkers, and chemical/biological sensors. ${ }^{11,12}$
Ag NPs can be synthesized using various methods, namely chemical reduction, ${ }^{13}$ electrochemistry, ${ }^{14}$ photochemistry, ${ }^{15}$ laser ablation, ${ }^{16}$ sonochemistry, ${ }^{17}$ and sputtering. ${ }^{18}$ Among these, the most popular method for the preparation of silver colloids is based on the chemical reduction of the metal ions by a reducing agent, usually the solvent, and generally in the presence of a stabilizing agent. Typical reducing agents include polyols. ${ }^{19}$ The polyol process is commonly used for the preparation of easily reducible metals. ${ }^{20}$ Polyols such as ethylene glycol (EG) can act as a reducing agent and as a solvent in which the metal salts are dissolved. The most commonly used stabilizing agents are polymers and surfactants. $^{21}$ Poly(vinylpyrrolidone) (PVP) is a widely used polymer to passivate the surface of silver nanoparticles and protect the nanoparticles from sintering. ${ }^{22}$ Polyol-mediated synthesis can be carried out at room temperature, but higher temperatures are generally used for higher reaction rates. ${ }^{20}$ To increase the reaction media temperature, conventional thermal heating $^{23}$ or ultrasonic irradiation ${ }^{24}$ can be employed. Many of

Received: November 5, 2019

Accepted: February 21, 2020

Published: March 10, 2020 

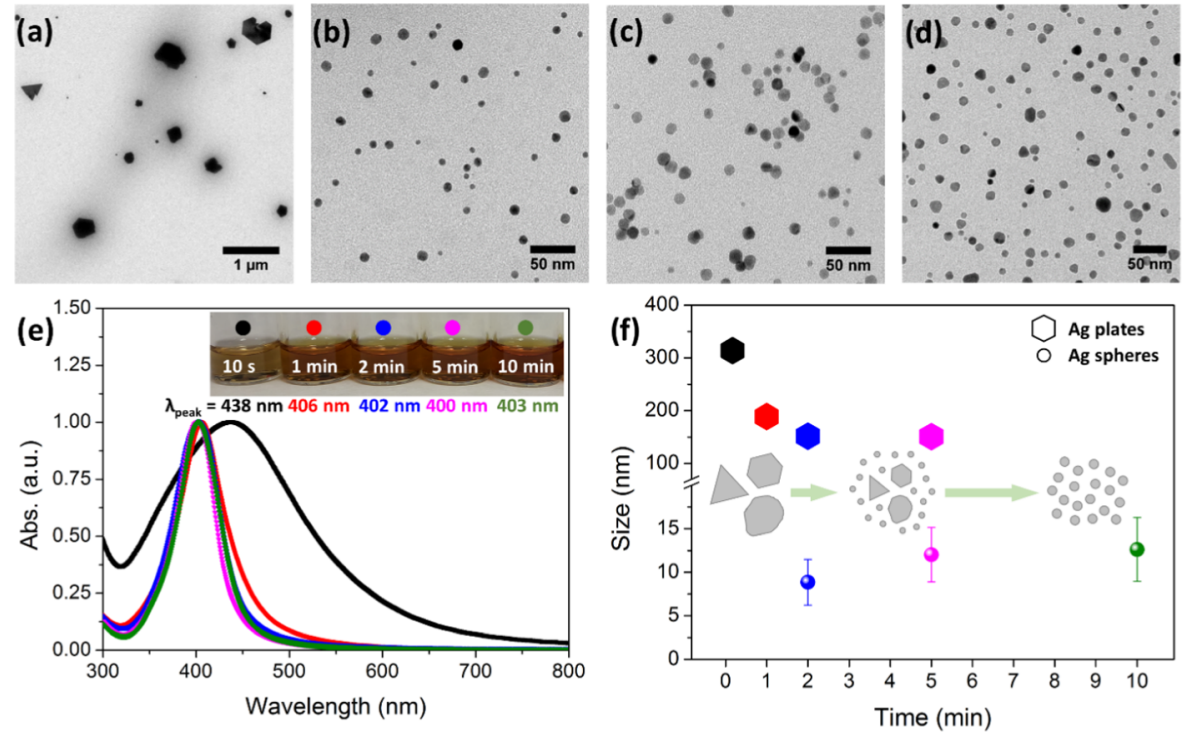

Figure 1. Effect of the reaction time (at $120^{\circ} \mathrm{C}$ and $1 \mathrm{mM} \mathrm{AgNO}_{3}$ ). (a-d) Representative TEM images of the different reaction times analyzed (10 $\mathrm{s}, 2 \mathrm{~min}, 5 \mathrm{~min}$, and $10 \mathrm{~min}$, respectively). (e) UV-vis measurements of the same samples. The inset contains digital images of the samples. (f) Mean size evolution with time (the error bars represent the standard deviation over the population mean). Inset: scheme of the Ag NP evolution with the reaction time.

these preparations are limited due to long reaction times, the consumption of large volumes of solvent, and poor stability of the products. Microwave (MW)-assisted chemistry is an advantageous heating source that provides volumetric uniform heating of the reaction solution (leading to narrow size distributions), shorter reaction times, lower energy consumption, and high product yield. ${ }^{25-27}$ Microwave ovens permit the synthesis of many types of inorganic nanoparticles, from single component (metals and metal oxides) ${ }^{28}$ to multicomponent nanoparticles or metastable alloys. ${ }^{29-31}$

One of the most powerful advantages of the MW technique is that it is rapid. ${ }^{25-27}$ Its fast heating velocity, combined with the possibility to thermally quench the reactions, enabled us to grasp snapshots of nucleation and growth processes difficult to accomplish in other techniques. Consequently, some mechanistic insights can be extracted as well as information on competing kinetics and thermodynamics processes, permitting the stabilization of metastable phases. These advantages can lead to a greater understanding of the key parameters to obtain a wide range of desired products, as well as to control the size and shape of NPs to yield desirable chemical and physical properties.

Here, we report a systematic and detailed study of the diverse scenarios of Ag NP synthesis mapping several synthetic conditions such as the reaction time, the reaction temperature, and the silver precursor concentration. Platelet-like kinetically favored Ag structures could be obtained for the first time using highly reducing conditions benefitting from the very short reaction times of the MW-assisted synthesis as opposed to thermodynamically favored morphologies such as spheres obtained at longer reaction times and higher temperatures. $^{32-35}$ A formation mechanism from silver polyhedral plates to spherical nanoparticles is proposed. Understanding these effects allowed us to control the particle size and the tuning of Ag NPs on-demand. Moreover, the reproducibility and scalability of the process were demonstrated. Finally, this study provides a recommendation regarding the use of PVP as a capping and stabilizing agent through a polyol approach.

\section{RESULTS AND DISCUSSION}

Fast synthesis of metal $(\mathrm{Au})$ and metal oxide $\left(\mathrm{Fe}_{2} \mathrm{O}_{3}, \mathrm{TiO}_{2}\right)$ nanoparticles by MW-assisted chemistry, as well as their combination in hybrid nanostructures, was previously reported by our group. ${ }^{29-31}$ Here, $\mathrm{Ag}$ nanoplates and nanoparticles are synthesized by a polyol approach using $\mathrm{AgNO}_{3}$ as the precursor, ethylene glycol (EG) as the solvent and the reducing agent, and polyvinylpyrrolidone (PVP) as the reducing and capping agent.

2.1. Effect of the Reaction Time. The effect of the reaction time on the resulting $\mathrm{Ag}$ NPs was investigated using 1 $\mathrm{mM} \mathrm{AgNO}, 120{ }^{\circ} \mathrm{C}$ reaction temperature, and synthesis times of $10 \mathrm{~s}, 1,2,5$, and $10 \mathrm{~min}$; the results are shown in Figure 1. Transmission electron microscopy (TEM) micrographs confirmed the successful formation of nanoparticles in all conditions and depict the differences in terms of particle size, shape, and polydispersity. For all particles, selected area electron diffraction (SAED) patterns confirmed a face-centered cubic metallic silver as the sole crystalline phase (Figure S1). At $10 \mathrm{~s}$, an extremely short reaction time, particles display various shapes, commonly polyhedral plates such as triangles and hexagons and mean sizes larger than $300 \mathrm{~nm}$ with a large polydispersity of sizes, as can be observed in Figure 1a. Similar results are obtained at $1 \mathrm{~min}$ reaction time, with a size reduction $(\sim 200 \mathrm{~nm})$. The particle size is further reduced at longer reaction times. At 2 min reaction time, the majority of the population consisted of small, spherical, and monodisperse NPs (PDI < 30\%) (Figure $1 \mathrm{~b}-\mathrm{d}$ ). Particles have a mean size of $9 \pm 3,12 \pm 3$, and $13 \pm 4 \mathrm{~nm}$ at 2,5 , and $10 \mathrm{~min}$, respectively, resulting from the fitting of the size histogram to a Gaussian distribution. A minority population of larger particles was also observed with mean sizes of $\sim 150 \mathrm{~nm}$ for 2 and 5 min reaction times. Thus, by increasing the reaction time from seconds to $10 \mathrm{~min}$, particles experience a size refinement where the initial nanoplates steadily disappear in favor of spherical monodispersed nanoparticles. Moreover, above $2 \mathrm{~min}$ of the reaction, the longer the reaction time, the bigger the particles. 

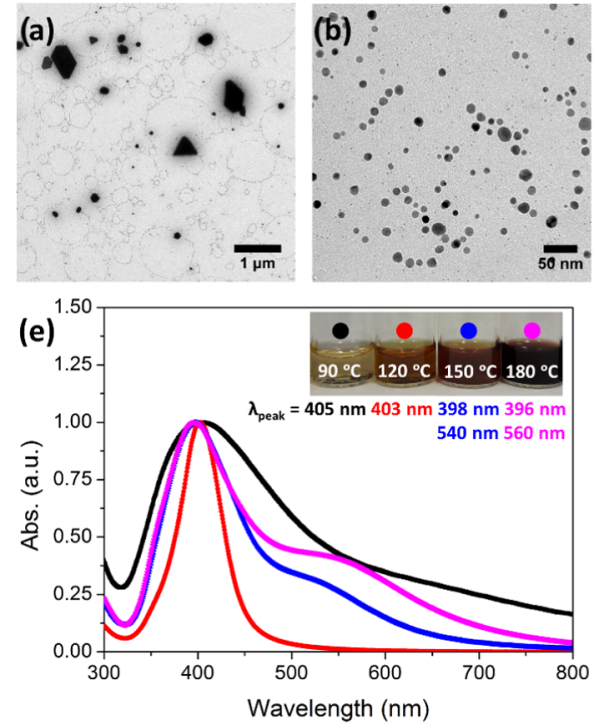
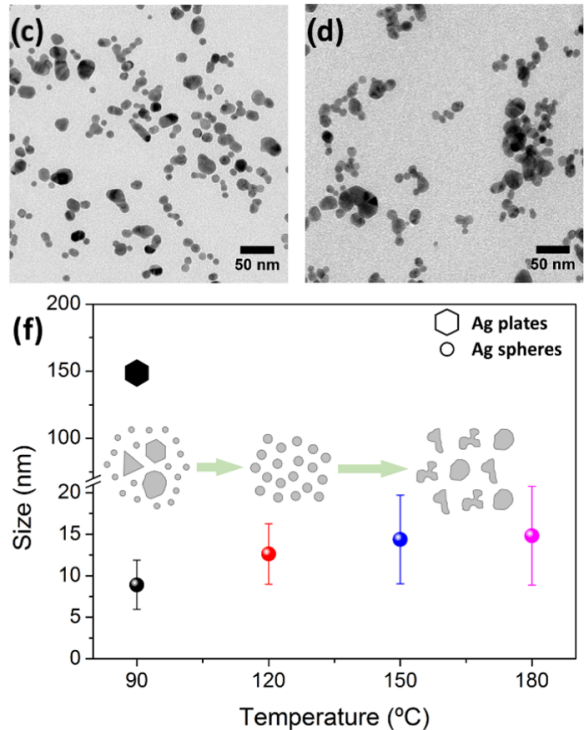

Figure 2. Effect of the reaction temperature (at $10 \mathrm{~min}$ and $1 \mathrm{mM} \mathrm{AgNO}_{3}$ ). (a-d) Representative TEM images of the different reaction temperatures analyzed $\left(90,120,150\right.$, and $180{ }^{\circ} \mathrm{C}$, respectively). (e) UV-vis measurements of the same samples. The inset contains digital images of the samples. (f) Mean size evolution with temperature (the error bars represent the standard deviation over the population mean). Inset: scheme of the Ag NP evolution with the reaction temperature.
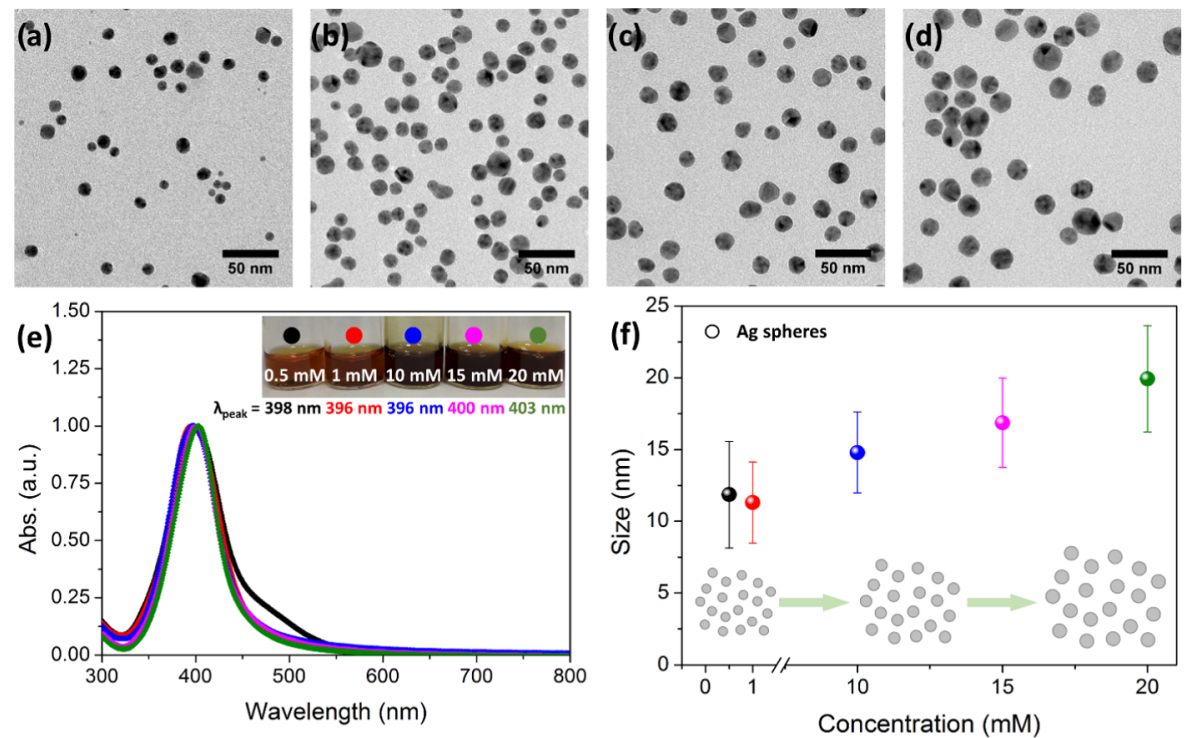

Figure 3. Effect of the reaction concentration (at $10 \mathrm{~min}$ and $\left.120^{\circ} \mathrm{C}\right) .(\mathrm{a}-\mathrm{d})$ Representative TEM images of the different reaction concentrations analyzed $(1,10,15$, and $20 \mathrm{mM}$, respectively). (e) UV-vis measurements of the same samples. The inset contains digital images of the samples. (f) Mean size evolution with concentration (the error bars represent the standard deviation over the population mean). Inset: scheme of the Ag NP evolution with the reaction concentration.

TEM observations were confirmed by UV-vis spectroscopy. Figure 1e shows the absorbance spectra for these materials. In all cases, one single peak corresponding to the localized surface plasmon resonance (LSPR) of Ag NPs is observed. It is well established that the shape, size, and composition of metal nanoparticles are crucial in determining the LSPR spectral position. $^{5-7}$ According to the previous size distributions, polydisperse samples of $10 \mathrm{~s}$ and $1 \mathrm{~min}$ have a broad LSPR peak, while monodisperse samples from 2, 5, and $10 \mathrm{~min}$ reaction times have a narrow one. Moreover, the wavelength of the maximum of the UV-vis bands shifted from $438 \mathrm{~nm}$ at 10 $\mathrm{s}$ to $403 \mathrm{~nm}$ at $10 \mathrm{~min}$, due to the smaller size and isotropic shapes. Figure 1f shows the mean sizes vs reaction times of $\mathrm{Ag}$ NPs.

2.2. Effect of the Reaction Temperature. The effect of the reaction temperature was investigated by using $1 \mathrm{mM}$ $\mathrm{AgNO}_{3}, 10 \mathrm{~min}$ reaction time, and synthesis temperatures of $60,90,120,150$, and $180{ }^{\circ} \mathrm{C}$, and the results are shown in Figure 2. At $60{ }^{\circ} \mathrm{C}$, a slight color change on the reaction tube was observed, but no precipitate was collected. This indicates that at this low temperature, no nucleation occurs. However, the tube changed its color to the expected orange-yellow after being stored one night, due to the room temperature reduction of silver cations for a longer time by EG and PVP acting as the 
reducing agents. Indeed, the chemical reduction reaction involved in this system is kinetically controlled.

When the temperature is increased to $90{ }^{\circ} \mathrm{C}$, NPs were formed after $10 \mathrm{~min}$ of the reaction. Figure $2 \mathrm{a}$ shows a representative TEM image of the sample, in which two populations are observed: a minority-bigger, polydisperse, with polyhedral shapes and with an average size of around 150 $\mathrm{nm}$; and a majority - smaller, monodisperse and spherical with $9 \pm 3 \mathrm{~nm}$ size. These results are almost the same as those found for the reaction time sample of $2 \mathrm{~min}$ (Figure 1b), heated at $120{ }^{\circ} \mathrm{C}$. It shows how the system heated at higher temperatures displays an accelerated reduction; therefore, in 2 min at $120^{\circ} \mathrm{C}$, the same particles are formed as in $10 \mathrm{~min}$ at 90 ${ }^{\circ} \mathrm{C}$.

At $120{ }^{\circ} \mathrm{C}$ (Figure $2 \mathrm{~b}$ ), the Ag NPs were identical to the sample reported in Figure 1d. Thus, the reproducibility of MW-assisted syntheses is demonstrated. On increasing the temperature further, the most significant observation was the gradual increase in the mean particle size and the loss of monodispersity. Specifically, particles have an average size of $14 \pm 5$ and $15 \pm 6 \mathrm{~nm}$ at 150 and $180{ }^{\circ} \mathrm{C}$, respectively. The loss of monodispersity is attributed to the fact that at higher temperatures, particles start to fuse; aggregates are clearly observed in Figure 2c,d.

UV-vis spectra for the samples of this series were recorded (Figure 2e). The sample at $90{ }^{\circ} \mathrm{C}$ has a broad LSPR peak at $405 \mathrm{~nm}$ wavelength, while the most monodisperse sample (120 ${ }^{\circ} \mathrm{C}$ ) has a narrower band with a maximum at $403 \mathrm{~nm}$. Otherwise, samples at higher temperatures $\left(150\right.$ and $180{ }^{\circ} \mathrm{C}$ ) show two broad peaks at 398 and $540 \mathrm{~nm}$ and at 396 and 560 $\mathrm{nm}$, respectively. These secondary and red-shifted bands correspond to the bigger and nonisotropic particles present in these samples in accordance with TEM results. Figure $2 \mathrm{f}$ shows the mean sizes vs reaction temperatures of Ag NPs.

2.3. Effect of the Silver Precursor Concentration. The effect of the silver precursor concentration was also investigated by using $10 \mathrm{~min}$ reaction time, $120{ }^{\circ} \mathrm{C}$ reaction temperature, and the synthesis concentrations of $0.5,1,10,15$, and $20 \mathrm{mM} \mathrm{AgNO}_{3}$; the results are shown in Figure 3. On increasing the concentration, the most significant observations were the gradual increase in the average size and the gradual increase in the number of nanoparticles (more particles were collected). Both phenomena are likely to occur simultaneously because of the increase of the number of silver nuclei in the initial solution (coming from the $\mathrm{Ag}(\mathrm{I})$ reduction) with higher concentrations. Particularly, particles have an average size determined by TEM of $12 \pm 4,11 \pm 3,15 \pm 3,17 \pm 3$, and 20 $\pm 4 \mathrm{~nm}$ at $0.5,1,10,15$, and $20 \mathrm{mM} \mathrm{AgNO}_{3}$, respectively. Then, in the range between 0.5 and $1 \mathrm{mM} \mathrm{AgNO}_{3}$, particles are spherical, monodisperse, and have a similar size of around $13 \mathrm{~nm}$; when the concentration is increased by 1 order of magnitude $\left(10,15\right.$, and $\left.20 \mathrm{mM} \mathrm{AgNO}_{3}\right)$, particles can grow up to 15,17 , or $20 \mathrm{~nm}$.

UV-vis spectra for the samples of this series were recorded (Figure 3e). The silver LSPR band maxima were located at $398,396,396,400$, and $403 \mathrm{~nm}$ wavelengths at $0.5,1,10,15$, and $20 \mathrm{mM} \mathrm{AgNO}_{3}$, respectively. Samples experience a red shift with concentration according to their bigger size, as was seen in the TEM images. Note that samples with more polydispersity have broader LSPR bands. Figure $3 \mathrm{f}$ shows mean sizes vs silver precursor concentrations of Ag NPs.

2.4. Additional Experiments. To study the influence of agitation, the concentration of PVP, and the initial solvent of the silver precursor, additional synthesis was also undertaken. The synthesis conditions were always $10 \mathrm{~min}$ reaction time, $120{ }^{\circ} \mathrm{C}$ reaction temperature, and $1 \mathrm{mM} \mathrm{AgNO}_{3}$. The influence of agitation was studied by heating the above solution in the MW reactor without agitation, the PVP concentration control was studied by changing the PVP amount from 25 to $5 \mathrm{mg}$, and the silver precursor solvent control was studied using EG instead of $\mathrm{MQ}-\mathrm{H}_{2} \mathrm{O}$ to prepare the initial $\mathrm{AgNO}_{3}$ solution.

When the reaction solution is not stirred during heating, the obtained Ag NPs are polydisperse, as shown in the TEM image (Figure 4a). Indeed, the corresponding UV-vis spectrum

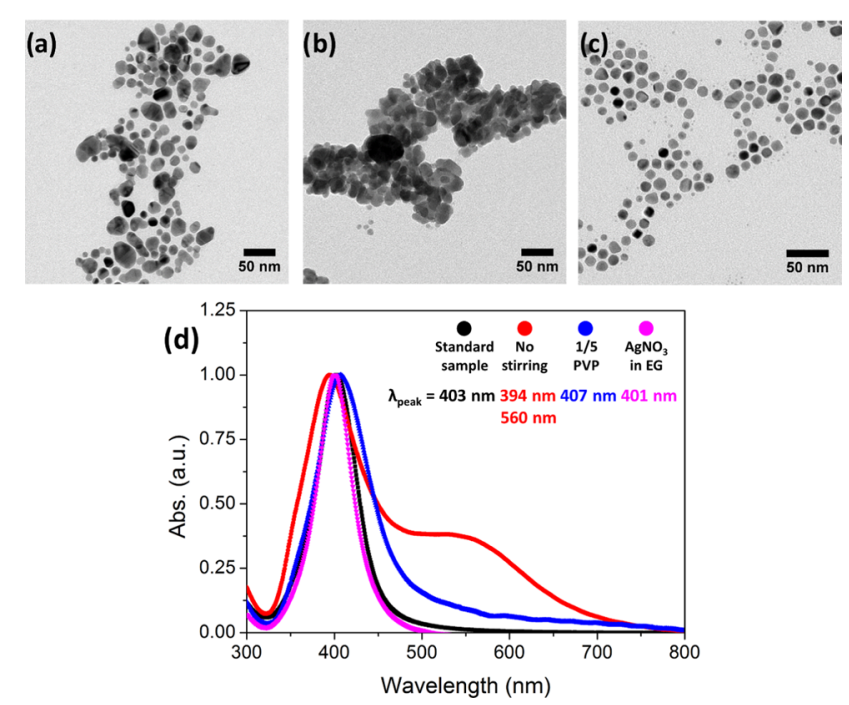

Figure 4. Control experiments (at $10 \mathrm{~min}, 120{ }^{\circ} \mathrm{C}$, and $1 \mathrm{mM}$ of $\left.\mathrm{AgNO}_{3}\right)$. $(\mathrm{a}-\mathrm{c})$ Representative TEM images of the different control assays analyzed (nonagitated reaction, five times less PVP and initial $\mathrm{AgNO}_{3}$ solution in EG, respectively). (d) UV-vis of the same samples. The standard sample $\left(10 \mathrm{~min}, 120^{\circ} \mathrm{C}, 1 \mathrm{mM} \mathrm{AgNO}{ }_{3}\right.$ from an aqueous solution, agitation, and $25 \mathrm{mg}$ of PVP) is also represented for comparison.

clearly defines two LSPR peaks at 394 and $560 \mathrm{~nm}$ wavelengths (Figure $4 d$ ). Agitation results in increased homogeneity of the final spherical Ag NPs because it avoids diffusion control of the growth process after new nucleation of $\operatorname{Ag}(0)$ elements. When the PVP concentration is reduced five times, $\mathrm{Ag}$ NP aggregates are observed, implying that there is not enough stabilizer both sterically and electrostatically (Figure $4 \mathrm{~b}$ ). Accordingly, the UV-vis spectrum of this sample shows a broad peak at $407 \mathrm{~nm}$ wavelength (Figure 4d). Finally, when EG is used to prepare the initial $\mathrm{AgNO}_{3}$ solution (instead of a small amount of water used in the described synthesis before), no significant differences were found. Ag NPs are spherical, monodisperse, and well stabilized by PVP (Figure 4c) and the corresponding $\mathrm{UV}$-vis spectrum supports this (narrow peak at $401 \mathrm{~nm}$ ) as also for the standard sample represented in Figure 4d (10 min, $120{ }^{\circ} \mathrm{C}, 1 \mathrm{mM} \mathrm{AgNO}_{3}$ from an aqueous solution, agitation, and $25 \mathrm{mg}$ of PVP).

2.5. Mechanistic Insights. After all of the experiments described above, some mechanistic insights can be hypothesized. Figure 5 schematizes a simplified mechanism proposed for the formation of the Ag NPs.

We have observed that the reaction starts with anisotropic platelet-like structures (triangles, hexagons, and truncated shapes), as shown for the samples at $10 \mathrm{~s}, 120^{\circ} \mathrm{C}$ and $10 \mathrm{~min}$, 


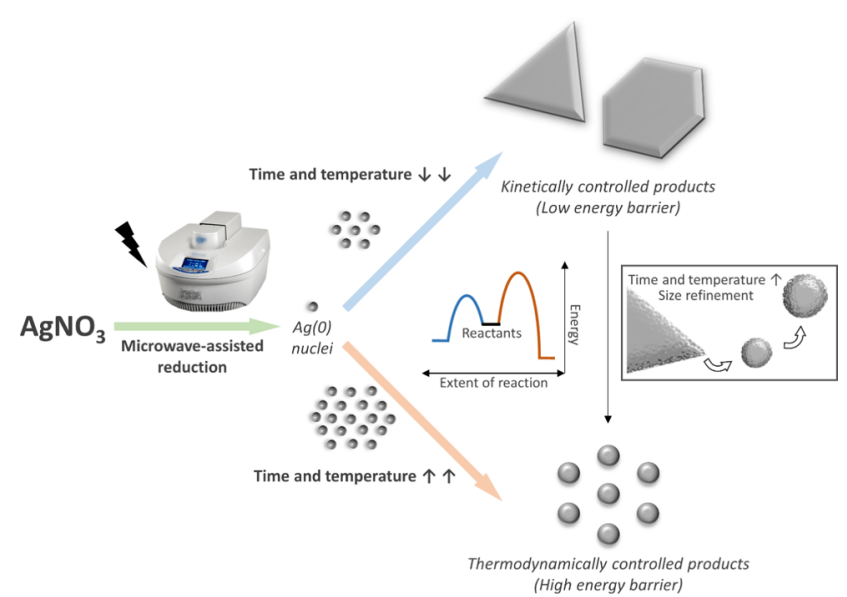

Figure 5. Schematic representation of the formation of Ag NPs. The proposed mechanism for the formation of $\mathrm{Ag}$, big, anisotropic nanostructure-like platelets and Ag, small, spherical NPs depending on the synthetic conditions. Notation: $\mathrm{AgNO}_{3}$, silver nitrate; $t$, time; $T$, temperature; PVP, polyvinylpyrrolidone.

$90{ }^{\circ} \mathrm{C}$ (Figures $1 \mathrm{a}$ and $2 \mathrm{a}$, respectively). When the reduction starts, $\mathrm{Ag}$ atoms are formed in the solution and tend to aggregate into small clusters or nuclei, which then aggregate into nanoparticles. ${ }^{36}$ If the reduction is fast (high temperature for a long time), there are enough $\mathrm{Ag}$ atoms to attach to the seeds' surface for continuous growth, tending to thermodynamically favored products. However, if the reduction is slow (low temperature or short times at high temperature and fast quenching), the concentration of $\mathrm{Ag}$ atoms is small, and the nanocrystals will take shapes deviating from the thermodynamically favored ones. ${ }^{37}$ This synthesis is kinetically controlled. In this case, stacking faults can develop and induce the formation of thin nanoplates despite their high total energy. ${ }^{19,38-40}$ Each $\mathrm{Ag}$ nanoplate is enclosed by two $\{111\}$ planes as the top and bottom faces, and a mix of $\{100\}$ and $\{111\}$ planes as the side faces. ${ }^{41}$ Moreover, these structures have $\{111\}$ twin planes parallel to their flat faces ${ }^{38}$ and stacking faults. ${ }^{7,42}$ Then, due to a large surface area and the lattice strain caused by stacking faults, the formation of nanoplates is not favored in terms of surface energy. For this reason, $\mathrm{Ag}$ nanoplates are kinetically favored products.

There are different methods of achieving kinetically controlled synthesis of metal nanoplates: the coupling of reduction with oxidative etching, ${ }^{43}$ the use of an extremely mild reducing agent, ${ }^{44}$ and the use of ligands to form complexes with $\operatorname{Ag}(\mathrm{I})$ ions. $^{38}$ In this work, note that MW heating of $\mathrm{AgNO}_{3}$ in EG in the presence of PVP causes the reduction of $\operatorname{Ag}(\mathrm{I})$ to $\operatorname{Ag}(0)$, where both EG and PVP act as reducing reagents. This together with the fast heating of the solution with the MW-assisted synthesis method allows the rapid formation of many $\mathrm{Ag}$ atoms that are able to grow into thermodynamically favored morphologies such as spheres (as it happens at long reaction times). However, thanks to the fast heating and cooling speeds of the MW method, we were able to capture and freeze kinetically favored products in conditions (extremely short reaction time or very low temperature) where a smaller number of silver nuclei were allowed to grow. Note that the heating and the cooling velocities are high (around 1 ${ }^{\circ} \mathrm{C} / \mathrm{s}$ ). Indeed, the energy barrier to achieving kinetically favored products is lower than the one for thermodynamic ones. Thus, the reaction starts with $\mathrm{Ag}$ nanoplates and it stabilizes with Ag nanospheres. Moreover, the PVP present during the reaction can help the formation of $\mathrm{Ag}$ nanoplates. When $\operatorname{Ag}(0)$ nucleates, free PVP adsorbs to all silver surfaces due to the strong affinity of the Ag surface to the nitrogen of the PVP, resulting in small, spherically shaped $\mathrm{Ag}$ NPs. However, decreasing the amount of PVP could break the initial symmetry to avoid spherical growth. During crystal growth, free PVP will preferentially adsorb to the faces with the lowest energy. Then, selective adsorption occurs on the $\{111\}$ faces (top and bottom faces of the Ag nanocrystals), while $\{100\}$ faces (three-sided faces) continued to grow at a faster rate. Thus, we expect that reducing further the PVP amount in the reaction media (without compromising the NP final stability and dispersion) could help to drive the nanoplate formation. Moreover, less PVP could lead to the large edge length of the observed anisotropic Ag nanostructure-like platelets (Figures $1 \mathrm{a}$ and $2 \mathrm{a}$ ).

Finally, increasing the reaction time and/or the reaction temperature drives the system to thermodynamically favored products, such as the spherical structures observed in Figures 1-4. Indeed, Ag nanoplates are thermodynamically unstable in comparison with Ag spherical particles. As a metal inside a conventional MW oven, the big silver nanoplates can act as an antenna $^{25,45}$ and absorb the MW irradiation, promoting its fracture to small particles. Then, particles undergo a size refinement, decreasing their size and changing their shape. In addition, at longer times, higher temperatures, and higher silver precursor concentration, particles increase their size (and number) due to the increased reaction that contributes to new nucleation and growth.

2.6. Ag NPs On-Demand, Scalability of the Protocol, and Reaction Yield. Comprehending all of these effects allowed us to control the particle size and the tuning of Ag NPs on demand (Figure 6). For instance, if bigger monodisperse Ag NPs are desired, time, concentration, and reaction temperature $\left(<150{ }^{\circ} \mathrm{C}\right)$ can be increased. A successful test was prepared with the synthesis conditions fixed to $20 \mathrm{~min}, 130{ }^{\circ} \mathrm{C}$, and 20

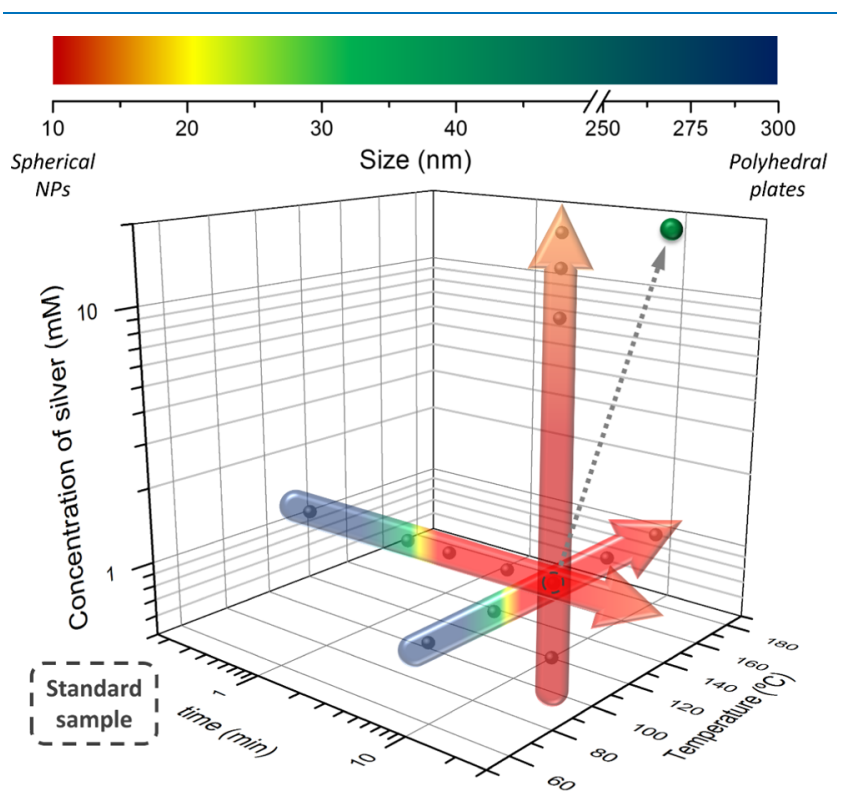

Figure 6. Summary of Ag NP mean sizes depending on the synthetic conditions. The single green dot corresponds to the planned synthesis to demonstrate the capability of producing Ag NP with on-demand sizes, $\sim 30 \mathrm{~nm}$ in this case. 
$\mathrm{mM} \mathrm{AgNO}$. The obtained Ag NPs have a mean size of $33 \pm$ $14 \mathrm{~nm}$ (Figure S2a and the single green dot in Figure 6). These particles are approx. $20 \mathrm{~nm}$ larger than the ones of the standard sample (Figure S2b). Consequently, a red shift was observed in their UV-vis spectrum (Figure S2c).

It is well known that MW-assisted synthesis can be easily scaled up, accelerating the migration of nanoparticles from the laboratory to the market. We show that the synthesis presented here can be scaled up at least by a factor of 4 . To study the scalability of the protocol, both, the same MW reactor (CEM) and a Microwave Advanced Flexible Synthesis Platform (flexiwave) from Milestone, were used. According to TEM images (Figure S3a,b), Ag NPs were successfully fabricated by scaling up the synthesis by a factor of 4 with both MW reactors, having an average size of $16 \pm 4$ and $24 \pm 4 \mathrm{~nm}$ when CEM and Flexiwave MW reactors were used, respectively. In comparison with the standard sample (Figure S3c), these spherical and always monodisperse Ag NPs are bigger. Therefore, a red shift was observed in their UV-vis spectrum (Figure S3d). Such slight increase of the particle size may come from the non-linear phenomena likely to occur when one synthesis is scaled up, which should be readjusted on demand (outside the scope of this study).

Finally, a chemical analysis of Ag was done with inductively coupled plasma optical emission spectroscopy (ICP-OES). The results showed a chemical reaction yield of $57 \%$ for the standard sample at CEM MW reactor, corresponding to 0.25 $\mathrm{mg}$ of Ag. On the other hand, when the same CEM MW reactor was used for the four-fold scale-up, the yield was $61 \%$ corresponding to $1.05 \mathrm{mg}$ of Ag per batch. Then, the defined MW-protocol and the cleaning steps of this work for the preparation of Ag NPs with controlled size result, on average, in a $60 \%$ yield and the production of silver colloids up to $1 \mathrm{mg}$. Higher quantities should be expected when using the Flexiwave MW reactor due to its greater scalability potential, since up to 15 reaction-vases of $100 \mathrm{ml}$ can be used in one batch.

\section{CONCLUSIONS}

We have presented a fast, simple, reproducible, and scalable microwave-assisted polyol synthesis of Ag NPs with different shapes and controlled sizes by a judicious choice of the synthetic parameters such as reaction time, reaction temperature, and silver precursor concentration. We have hypothesized mechanistic insights for the formation of the obtained nanoplates and nanoparticles underpinned by the observations extracted from transmission electron microscopy (TEM) and ultraviolet-visible spectroscopy (UV-vis).

Very short reaction times and low temperatures lead to a small number of available nuclei yielding to kinetically favorable anisotropic platelet-like structures (triangles, hexagons, and truncated shapes). Moreover, the low amounts of PVP used facilitate breaking the initial spherical symmetry by preferential adsorption to the $\{111\}$ faces (top and bottom faces of Ag nanocrystals), while $\{100\}$ faces (three-sided faces) continue to grow at a faster rate, leading to the anisotropic $\mathrm{Ag}$ platelet-like structures. Note that platelet structures were observed here for the first time in synthetic conditions (EG and PVP allow high reduction rates), where for longer reaction times and higher temperatures, there is a rapid formation of a large number of $\mathrm{Ag}$ nuclei that grow into thermodynamically favored morphologies such as spheres. Indeed, Ag nanoplates are thermodynamically unstable in comparison with $\mathrm{Ag}$ spherical particles. Then, particles undergo a size refinement, decreasing their size and changing their shape. In addition, at longer times, higher temperatures, and higher silver precursor concentrations, particles increase their size (and number) due to the increased reaction that contributes to new nucleation and growth.

Understanding these effects allowed us to control the particle size and the tuning of Ag NPs on-demand using cross experiments. Moreover, the reproducibility and scalability of the process and the long-term stability -up to 9 months- of the NPs in aqueous solutions was demonstrated. Finally, we provide a recommendation regarding the use of fresh PVP as a capping and stabilizing agent to reproduce the nanoparticles' synthetic results (see the Experimental Section).

\section{EXPERIMENTAL SECTION}

4.1. Materials. Silver nitrate for analysis, ACS, ISO $\left(\mathrm{AgNO}_{3}, 99.8 \%\right)$, ethylene glycol (EG $\left.\geq 99 \%\right)$, and acetone were purchased from Panreac. Poly(vinylpyrrolidone) (PVP, average molecular weight: $10000 \mathrm{~g} / \mathrm{mol}$ ) was purchased from Sigma-Aldrich. All materials were used as received without further purification. Milli-Q water $\left(\mathrm{MQ}-\mathrm{H}_{2} \mathrm{O}\right)$ was used in all experiments.

4.2. Synthesis of PVP-Coated Ag NPs. Ag NPs with a PVP surface coating are synthesized using a microwave (MW)assisted polyol synthesis protocol in a CEM Discover SP (with the autosampler Explorer 12 Hybrid) at a frequency of 2.45 $\mathrm{GHz}$ and $300 \mathrm{~W}$ of power. This MW reactor is equipped with pressurized vessels to which an active cooling with compressed air permits to control the set reaction temperature as well as to rapidly quench the reaction at the end of the set time preventing Ostwald ripening. Note that since the reaction vessel remains at room temperature during the reaction, this quenching mechanism is very effective.

Briefly, $25 \mathrm{mg}$ of PVP $(2.5 \mu \mathrm{mol})$ is dissolved in $4 \mathrm{ml}$ of EG by continuous sonication (JP Selecta 3000683, frequency, 40 $\mathrm{kHz}$; sonication time, $5 \mathrm{~min}$ ). Then, $4 \mu \mathrm{mol}$ of $\mathrm{AgNO}_{3}$ (from an aqueous solution, $250 \mathrm{mM}$ ) is mixed with the aboveprepared solution to give a homogeneous solution with no color of $1 \mathrm{mM} \mathrm{AgNO}_{3}$. The tubes (CEM, borosilicate glass, and silicon with septum caps) are then placed in the MW reactor and heated under continuous agitation (Labbox, $3 \times 6$ $\mathrm{mm}^{2}$ cylindrical, fully encapsulated with high-grade poly(tetrafluoroethylene) [PTFE-Teflon], and a maximum stirring speed of the CEM MW reactor). Heating and cooling velocities of the MW reactor were the same in all samples, with 1.1 and $0.8{ }^{\circ} \mathrm{C} / \mathrm{s}$, respectively. The effect of MW time $(t=$ $10 \mathrm{~s}, 1,2,5$, and $10 \mathrm{~min})$ and temperature $(T=60,90,120$, 150 , and $180{ }^{\circ} \mathrm{C}$ ) on the resulting silver structures was investigated. The effect of silver nitrate concentration $(0.5,1$, 10,15 , and $20 \mathrm{mM}$ ) was also studied. In this synthesis, PVP is used as the capping agent to shape and stabilize the Ag NPs and as a mild reducing agent, while EG acts as a solvent and a reducing agent. The final solution is orange-yellow, and no precipitate is observed, indicating that the nanoparticles are dispersed in the solution. Ag NPs are collected by adding 30 $\mathrm{ml}$ of acetone in the solution and centrifuging at $6000 \mathrm{rpm}$ for $30 \mathrm{~min}$ to precipitate the nanoparticles (Hettich EBA 21, type 1004, $4025 \mathrm{RCF})$. The supernatant with excess reactants (PVP, EG, $\mathrm{NO}_{3}{ }^{-}$, and $\mathrm{Ag}^{+}$ions not reduced) was discarded. The same procedure was repeated twice and the final solid NP precipitate was dispersed in $1 \mathrm{~mL}$ of $\mathrm{MQ}-\mathrm{H}_{2} \mathrm{O}$ and kept for further characterization. All of the above experiments were 
carried out in duplicate. PVP-capped Ag NPs are stable in water. Sample colloidal stability in water was demonstrated up to 9 months. Aggregates or precipitates were not significantly observed (Figure S4a). In addition, TEM analysis demonstrates their shape stability (Figure $S 4 b-g$ ).

Experiments to study the influence of agitation, PVP concentration, and initial conditions of silver nitrate were also prepared. Comprehending all these effects allowed us to control the particle size and tuning of Ag NPs on-demand.

This synthetic route can be scaled up by at least a factor of 4 . To study the scalability of the protocol, both the same MW reactor and a Microwave Advanced Flexible Synthesis Platform (flexiwave) from Milestone at a frequency of $2.45 \mathrm{GHz}$ and $500 \mathrm{~W}$ power were used.

We provide a recommendation regarding the use of fresh PVP as a capping and stabilizing agent. As mentioned above, all of the experiments were carried out in duplicate. The replicates were synthesized 4 months apart. In these samples, the behaviors found for both the controls and different effects were reproduced, demonstrating the robustness of the MWassisted synthesis. However, the resulting Ag NPs changed the morphology and a slight decrease in the average size was observed (Figure S5). These differences were attributed to PVP aging since, when new PVP was bought and used, the initial shapes were recovered. PVP polymer powder, according to the Safety Data Sheet (SDS), is stable and can be stored under ordinary conditions without undergoing decomposition or degradation. However, the powder is hygroscopic, forms molecular adducts with many other substances, and cross-links at high temperatures. This can result in a solubilizing action in some cases or in precipitation in others (in water or alcohol). Thus, all these potential uncontrolled changes of the PVP can affect the nanoparticles' synthetic results. Our final suggestion is to buy small batches of PVP and use them for no longer than 4 months.

4.3. Material Characterization. The morphologies and the crystalline phase of the Ag NPs were analyzed in a JEOL JEM-1210 transmission electron microscope (TEM) operating at $120 \mathrm{kV}$ and in a FEI Tecnai $\mathrm{G}^{2}$ F20 high-resolution transmission electron microscope (HRTEM) operating at 200 $\mathrm{kV}$. One drop of the NP dispersion was placed in a TEM grid (Micro to Nano, EMR Carbon support film on copper 200 square mesh). Typically, at least 500 different nanoparticles were measured with ImageJ to depict the size histogram. The mean particle size and standard deviation resulted from adjusting the particle size histograms to a Gaussian distribution function. An analysis of variance (ANOVA) test is used to successfully prove the significant differences in the mean particle size change by increasing the time, the temperature, and the concentration. In all cases, the $P$-value determined is $<0.0001$. Ultraviolet-visible (UV-vis) spectra were collected on a Varian Cary-5 UV-vis spectrophotometer between 200 and $800 \mathrm{~nm}$. An aliquot of the Ag NP dispersion is directly placed to the UV-vis cuvette and the obtained spectra normalized to the maximum absorbance value of the LSPR of the Ag NPs. Chemical analysis of Ag was done with inductively coupled plasma optical emission spectroscopy (ICP-OES) with an ICP-OES Perkin-Elmer, model Optima 4300DV. The samples were previously digested with concentrated nitric acid (for analysis, ISO, $\mathrm{HNO}_{3}, 65 \%$ ) in an ultrasound bath. Samples were carried out in duplicate. The reaction yield was calculated from these results.

\section{ASSOCIATED CONTENT}

\section{Supporting Information}

The Supporting Information is available free of charge at https://pubs.acs.org/doi/10.1021/acsomega.9b03748.

Diffraction data; size-control synthesis; high-resolution TEM images; scalability; colloidal and shape stability of Ag solution with time; effect of aging PVP on NP morphology (PDF)

\section{AUTHOR INFORMATION}

\section{Corresponding Author}

Anna Roig - Institut de Ciencia de Materials de Barcelona (ICMAB), CSIC 08193 Bellaterra, Spain; 이 orcid.org/00000001-6464-7573; Email: roig@icmab.es

\section{Author}

Miquel Torras - Institut de Ciencia de Materials de Barcelona (ICMAB), CSIC 08193 Bellaterra, Spain; 이이이.org/00000001-7132-6217

Complete contact information is available at:

https://pubs.acs.org/10.1021/acsomega.9b03748

\section{Notes}

The authors declare no competing financial interest.

\section{ACKNOWLEDGMENTS}

This research received funding from the Spanish Ministry of Science, Innovation and Universities through the PCIN-2017090 and RTI2018-096273-B-I00 projects, the "Severo Ochoa" Programme for Centers of Excellence in R\&D grants (SEV2015-0496), and the Generalitat de Catalunya (2017SGR765 grant). The Spanish Ministry of Education funded the FPU Fellow of MT (FPU16/05452). The authors would also like to thank Judith Oró from the TEM service at ICMAB and Marcos Rosado from the HRTEM service at ICN2 (Catalan Institute of Nanoscience and Nanotechnology).

\section{REFERENCES}

(1) Whitman, L. J.; Henderson, L. A.; Meador, M. A.; Friedersdorf, L. E.; Standridge, S.; Thomas, T.; Howard, J.; Biaggi-Labiosa, A. M.; Madsen, L.; Cannizzaro, C. et al. National Nanotechnology Initiative Strategic Plan; National Science and Technology Council, 2016.

(2) Yang, P.; Zheng, J.; Xu, Y.; Zhang, Q.; Jiang, L. Colloidal Synthesis and Applications of Plasmonic Metal Nanoparticles. Adv. Mater. 2016, 28, 10508-10517.

(3) Liu, L.; Zhang, X.; Yang, L.; Ren, L.; Wang, D.; Ye, J. Metal Nanoparticles Induced Photocatalysis. Natl. Sci. Rev. 2017, 4, 761780.

(4) Wu, X.; Hao, C.; Kumar, J.; Kuang, H.; Kotov, N. A.; LizMarzán, L. M.; Xu, C. Environmentally Responsive Plasmonic Nanoassemblies for Biosensing. Chem. Soc. Rev. 2018, 47, 4677-4696.

(5) Bastús, N. G.; Comenge, J.; Puntes, V. Kinetically Controlled Seeded Growth Synthesis of Citrate-Stabilized Gold Nanoparticles of up to $200 \mathrm{Nm}$ : Size Focusing versus Ostwald Ripening. Langmuir 2011, 27, 11098-11105.

(6) Duchene, J. S.; Niu, W.; Abendroth, J. M.; Sun, Q.; Zhao, W.; Huo, F.; Wei, W. D. Halide Anions as Shape-Directing Agents for Obtaining High-Quality Anisotropic Gold Nanostructures. Chem. Mater. 2013, 25, 1392-1399.

(7) Xia, Y.; Xiong, Y.; Lim, B.; Skrabalak, S. E. Shape-Controlled Synthesis of Metal Nanocrystals: Simple Chemistry Meets Complex Physics? Angew. Chem., Int. Ed. 2009, 48, 60-103. 
(8) Sharma, V. K.; Yngard, R. A.; Lin, Y. Silver Nanoparticles: Green Synthesis and Their Antimicrobial Activities. Adv. Colloid Interface Sci. 2009, 145, 83-96.

(9) Apalangya, V.; Rangari, V.; Tiimob, B.; Jeelani, S.; Samuel, T. Development of Antimicrobial Water Filtration Hybrid Material from Bio Source Calcium Carbonate and Silver Nanoparticles. Appl. Surf. Sci. 2014, 295, 108-114.

(10) Wei, L.; Lu, J.; Xu, H.; Patel, A.; Chen, Z. S.; Chen, G. Silver Nanoparticles: Synthesis, Properties, and Therapeutic Applications. Drug Discovery Today 2015, 20 (5), 595-601.

(11) Alshehri, A. H.; Jakubowska, M.; Młoziniak, A.; Horaczek, M.; Rudka, D.; Free, C.; Carey, J. D. Enhanced Electrical Conductivity of Silver Nanoparticles for High Frequency Electronic Applications. ACS Appl. Mater. Interfaces 2012, 4, 7007-7010.

(12) Natsuki, J. A Review of Silver Nanoparticles: Synthesis Methods, Properties and Applications. Int. J. Mater. Sci. Appl. 2016, $4,325$.

(13) Khan, Z.; Al-Thabaiti, S. A.; Obaid, A. Y.; Al-Youbi, A. O. Preparation and Characterization of Silver Nanoparticles by Chemical Reduction Method. Colloids Surf., B 2011, 82, 513-517.

(14) Rabinal, M. K.; Kalasad, M. N.; Praveenkumar, K.; Bharadi, V. R.; Bhikshavartimath, A. M. Electrochemical Synthesis and Optical Properties of Organically Capped Silver Nanoparticles. J. Alloys Compd. 2013, 562, 43-47.

(15) Maretti, L.; Billone, P. S.; Liu, Y.; Scaiano, J. C. Facile Photochemical Synthesis and Characterization of Highly Fluorescent Silver Nanoparticles. J. Am. Chem. Soc. 2009, 131, 13972-13980.

(16) Boutinguiza, M.; Comesaña, R.; Lusquiños, F.; Riveiro, A.; Del Val, J.; Pou, J. Production of Silver Nanoparticles by Laser Ablation in Open Air. Appl. Surf. Sci. 2015, 336, 108-111.

(17) Darroudi, M.; Khorsand Zak, A.; Muhamad, M. R.; Huang, N. M.; Hakimi, M. Green Synthesis of Colloidal Silver Nanoparticles by Sonochemical Method. Mater. Lett. 2012, 66, 117-120.

(18) Asanithi, P.; Chaiyakun, S.; Limsuwan, P. Growth of Silver Nanoparticles by DC Magnetron Sputtering. J. Nanomater. 2012, 2012, 1-8.

(19) Rodrigues, T. S.; Zhao, M.; Yang, T. H.; Gilroy, K. D.; da Silva, A. G. M.; Camargo, P. H. C.; Xia, Y. Synthesis of Colloidal Metal Nanocrystals: A Comprehensive Review on the Reductants. Chem. Eur. J. 2018, 24, 16944-16963.

(20) Fiévet, F.; Ammar-Merah, S.; Brayner, R.; Chau, F.; Giraud, M.; Mammeri, F.; Peron, J.; Piquemal, J. Y.; Sicard, L.; Viau, G. The Polyol Process: A Unique Method for Easy Access to Metal Nanoparticles with Tailored Sizes, Shapes and Compositions. Chem. Soc. Rev. 2018, 47, 5187-5233.

(21) Kang, H.; Buchman, J. T.; Rodriguez, R. S.; Ring, H. L.; He, J.; Bantz, K. C.; Haynes, C. L. Stabilization of Silver and Gold Nanoparticles: Preservation and Improvement of Plasmonic Functionalities. Chem. Rev. 2019, 119, 664-699.

(22) Huynh, K. A.; Chen, K. L. Aggregation Kinetics of Citrate and Polyvinylpyrrolidone Coated Silver Nanoparticles in Monovalent and Divalent Electrolyte Solutions. Environ. Sci. Technol. 2011, 45, 55645571.

(23) Zhao, T.; Sun, R.; Yu, S.; Zhang, Z.; Zhou, L.; Huang, H.; Du, R. Size-Controlled Preparation of Silver Nanoparticles by a Modified Polyol Method. Colloids Surf., A 2010, 366, 197-202.

(24) Byeon, J. H.; Kim, Y. W. A Novel Polyol Method to Synthesize Colloidal Silver Nanoparticles by Ultrasonic Irradiation. Ultrason. Sonochem. 2012, 19, 209-215.

(25) Bilecka, I.; Niederberger, M. Microwave Chemistry for Inorganic Nanomaterials Synthesis. Nanoscale 2010, 2, 1358-1374.

(26) Baghbanzadeh, M.; Carbone, L.; Cozzoli, P. D.; Kappe, C. O. Microwave-Assisted Synthesis of Colloidal Inorganic Nanocrystals. Angew. Chem., Int. Ed. 2011, 50, 11312-11359.

(27) Zhu, Y.-J.; Chen, F. Microwave-Assisted Preparation of Inorganic Nanostructures in Liquid Phase. Chem. Rev. 2014, 114, 6462-6555.

(28) Pascu, O.; Carenza, E.; Gich, M.; Estradé, S.; Peiró, F.; Herranz, G.; Roig, A. Surface Reactivity of Iron Oxide Nanoparticles by
Microwave-Assisted Synthesis; Comparison with the Thermal Decomposition Route. J. Phys. Chem. C 2012, 116, 15108-15116.

(29) Yu, S.; Hachtel, J. A.; Chisholm, M. F.; Pantelides, S. T.; Laromaine, A.; Roig, A. Magnetic Gold Nanotriangles by MicrowaveAssisted Polyol Synthesis. Nanoscale 2015, 7, 14039-14046.

(30) Hachtel, J. A.; Yu, S.; Lupini, A. R.; Pantelides, S. T.; Gich, M.; Laromaine, A.; Roig, A. Gold Nanotriangles Decorated with Superparamagnetic Iron Oxide Nanoparticles: A Compositional and Microstructural Study. Faraday Discuss. 2016, 191, 215-227.

(31) May-Masnou, A.; Soler, L.; Torras, M.; Salles, P.; Llorca, J.; Roig, A. Fast and Simple Microwave Synthesis of $\mathrm{TiO} 2 / \mathrm{Au}$ Nanoparticles for Gas-Phase Photocatalytic Hydrogen Generation. Front. Chem. 2018, 6, No. 110.

(32) Tsuji, M.; Hashimoto, M.; Nishizawa, Y.; Kubokawa, M.; Tsuji, T. Microwave-Assisted Synthesis of Metallic Nanostructures in Solution. Chem. - Eur. J. 2005, 11, 440-452.

(33) Jiang, H.; Moon, K. S.; Zhang, Z.; Pothukuchi, S.; Wong, C. P. Variable Frequency Microwave Synthesis of Silver Nanoparticles. J. Nanopart. Res. 2006, 8, 117-124.

(34) Tsuji, M.; Nishizawa, Y.; Matsumoto, K.; Miyamae, N.; Tsuji, T.; Zhang, X. Rapid Synthesis of Silver Nanostructures by Using Microwave-Polyol Method with the Assistance of Pt Seeds and Polyvinylpyrrolidone. Colloids Surf., A 2007, 293, 185-194.

(35) Dzido, G.; Markowski, P.; Małachowska-Jutsz, A.; Prusik, K.; Jarzębski, A. B. Rapid Continuous Microwave-Assisted Synthesis of Silver Nanoparticles to Achieve Very High Productivity and Full Yield: From Mechanistic Study to Optimal Fabrication Strategy. J. Nanopart. Res. 2015, 17, No. 27.

(36) Besson, C.; Finney, E. E.; Finke, R. G. A Mechanism for Transition-Metal Nanoparticle Self-Assembly. J. Am. Chem. Soc. 2005, $127,8179-8184$.

(37) Watzky, M. A.; Finke, R. G. Transition Metal Nanocluster Formation Kinetic and Mechanistic Studies. A New Mechanism When Hydrogen Is the Reductant: Slow, Continuous Nucleation and Fast Autocatalytic Surface Growth. J. Am. Chem. Soc. 1997, 119, 10382-10400.

(38) Xiong, Y.; Siekkinen, A. R.; Wang, J.; Yin, Y.; Kim, M. J.; Xia, Y. Synthesis of Silver Nanoplates at High Yields by Slowing down the Polyol Reduction of Silver Nitrate with Polyacrylamide. J. Mater. Chem. 2007, 17, 2600-2602.

(39) Pastoriza-Santos, I.; Liz-Marzán, L. M. Synthesis of Silver Nanoprisms in DMF. Nano Lett. 2002, 2, 903-905.

(40) Darmanin, T.; Nativo, P.; Gilliland, D.; Ceccone, G.; Pascual, C.; De Berardis, B.; Guittard, F.; Rossi, F. Microwave-Assisted Synthesis of Silver Nanoprisms/Nanoplates Using a "Modified Polyol Process”. Colloids Surf., A 2012, 395, 145-151.

(41) Wang, Z. L. Transmission Electron Microscopy of ShapeControlled Nanocrystals and Their Assemblies. J. Phys. Chem. B 2002, 104, 1153-1175.

(42) Wang, Y.; Peng, H. C.; Liu, J.; Huang, C. Z.; Xia, Y. Use of Reduction Rate as a Quantitative Knob for Controlling the Twin Structure and Shape of Palladium Nanocrystals. Nano Lett. 2015, 15, $1445-1450$

(43) Xiong, Y.; McLellan, J. M.; Chen, J.; Yin, Y.; Li, Z. Y.; Xia, Y. Kinetically Controlled Synthesis of Triangular and Hexagonal Nanoplates of Palladium and Their SPR/SERS Properties. J. Am. Chem. Soc. 2005, 127, 17118-17127.

(44) Washio, I.; Xiong, Y.; Yin, Y.; Xia, Y. Reduction by the End Groups of Poly(Vinyl Pyrrolidone): A New and Versatile Route to the Kinetically Controlled Synthesis of Ag Triangular Nanoplates. Adv. Mater. 2006, 18, 1745-1749.

(45) Dahal, N.; García, S.; Zhou, J.; Humphrey, S. M. Beneficial Effects of Microwave-Assisted Heating versus Conventional Heating in Noble Metal Nanoparticle Synthesis. ACS Nano 2012, 6, 94339446. 